\title{
EL CASTIGO DEL TIRANO (ALC. 143 VOIGT)
}

HELENA RODRIGUEZ SOMOLINOS

UNED

Un deteriorado fragmento de Alceo (Fr. 143 V.) parece hablarnos de una barbilla, de ceniza rociada y de un $\mu \delta \sigma \lambda \eta \bar{\zeta}$, término que en Sapph. 39.2 denomina un tipo de calzado, pero puede designar también una correa de cuero:

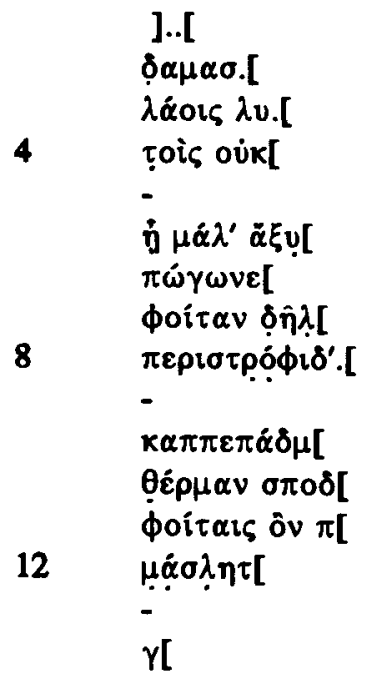


Según la inteligente interpretación de $\mathrm{W}$. Barner ${ }^{1}$, prácticamente la única que se ha intentado, el fragmento presenta primero una exhortación a luchar contra el tirano, después la descripción de los castigos que se le deben infligir: arrancarle la barba, hacerle mover la rueda de molino, rociarlo de ceniza caliente y azotarlo. Dichos castigos irían enumerados en infinitivos (como фoú$\tau \alpha v$ ) dependientes del adjetivo $\gamma \xi_{!}[0 \zeta]$, que Barner reconstruye en el verso $5^{2}$. El fragmento 388 Page de Anacreonte constituye el principal apoyo a esta reconstrucción, al enumerar las torturas sufridas en otro tiempo por un personaje que después prosperó, entre ellas arrancarle la barba y azotarlo. Por otro lado se compara este fragmento con Archil. 79 Diehl = Hippon. 115 West y Adrados, como vemos de atribución discutida, en que se piden todo tipo de desgracias contra un perjuro.

El punto más frágil de esta argumentación es en mi opinión la interpretación de $\pi \varepsilon \rho 1 \sigma \tau \rho o ̣ \phi 1 \delta$, cuestión en que no parece haber común acuerdo ${ }^{3}$. La pa-

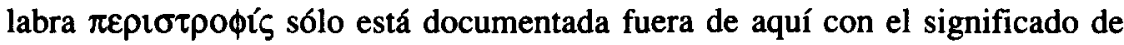
puño de la rueda de molino en Poll. 10.135 y con el de utensilio de madera que gira, rasero en Poll. 4.170 y 10.113. Como se puede ver, Barner toma el primero de estos dos significados para el término alcaico, lo cual no es del todo convincente. Según él como castigo el hombre haría girar la rueda de molino agarrándola del puño y andando ( $\phi o i \tau \alpha v)$ en círculo ${ }^{4}$. La primera objeción a esta idea está en que faltan otros testimonios para la tortura consistente en mover la rueda de molino. La comparación de Barner con el fragmento 389 de Alceo

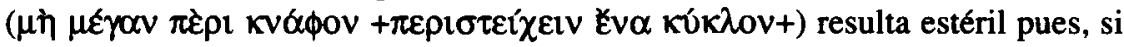
nos atenemos a sus fuentes, no se refiere a ninguna situación similar, y en cualquier caso el texto está demasiado corrupto para extraer conclusiones ${ }^{5}$. Por otro lado, los sentidos posibles del término son múltiples, tantos como nociones posee el verbo $\sigma \tau \rho \varepsilon \phi \omega$ (girar, girar sobre sí mismo, rodear, retorcer, etc.) ${ }^{6}$. El

${ }^{1}$ Neue Alkaios-Papyri aus Oxyrrhinchus. Spudasmata XIV, Tubinga-Hildesheim 1967, p. 30 ss.

2 En general se acepta la conjetura de Lobel $\measuredangle \xi \zeta[\rho \circ \zeta]$ sin afeitar, adjetivo conocido fuera de

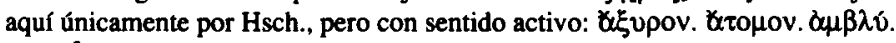

${ }^{3}$ El Suplemento a LSJ, Oxford 1968, lo considera de sentido dudoso.

4 Op. cit., p. 35: «der Mann soll zur Strafe die Walkermühle drehen (wobei er den Griff fasst und im Kreise geht; in der Lücke stand vielleicht eine Form wie

${ }^{5} \mathrm{La}$ interpretación de $\mathrm{kvó} \varphi \mathrm{\varphi}$, normalmente cardencha, como instrumento de tortura en Alceo procede de C. MASTrelli, La lingua di Alceo, Florencia 1954, p. 39, pero como digo va contra el testimonio de las fuentes. Por otro lado dicho fragmento 389 ha sido también atribuido al comico Alceo (Fr. 35 Kassel-Austin).

6 Precisamente Hamm, Grammatik zu Sappho und Alkaios. Abh. Berl. 2, 1957, p. 53. \$111c puso en relación nuestro término con $\pi \varepsilon \rho ı \tau \rho \circ \varphi \dagger(k \sigma \tau \rho \omega v)$ el curso de los astros en S. Fr. 432.8,

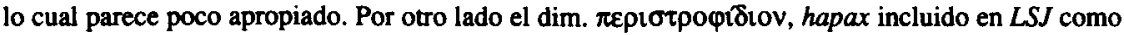
nombre de una prenda de vestir en una inscripción de Delos ( $B C H 29,1905$, p. 432, IV a.C.), que 
sentido de puño de la rueda de molino resulta excesivamente técnico, y el hecho de estar documentado sólo en Pólux hace poco probable que sea el mismo que utilizó Alceo. Contra esto se podría argumentar que Pólux recoge otros términos alcaicos raros ${ }^{7}$, pero a diferencia de lo que sucede con $\pi \varepsilon \rho 1 \sigma \tau \rho o \phi i \zeta$, en todos estos casos atribuye dichas glosas al poeta.

En nuestra opinión se hace necesario buscar un significado distinto, para lo cual creo que es oportuno seguir el camino iniciado por Mastrelli en su libro sobre la lengua de $\mathrm{Alceo}^{8}$.

En su glosario de términos alcaicos, Mastrelli presenta la división de palabras $\pi \varepsilon ́ p \imath \sigma \tau \rho \delta \phi 1 \delta^{\prime}$, considerando que este último término tiene el mismo significado que $\sigma \tau \rho o \phi i ́ \varsigma$ en E. Andr. 718, una banda o cinta. Sin embargo, esta lectura no carece de problemas, aludidos por Barner antes de exponer su interpretación.

En primer lugar, no se ve muy bien qué función podría tener dicha banda en medio de una descripción de torturas, aun dejando de lado el hecho de que

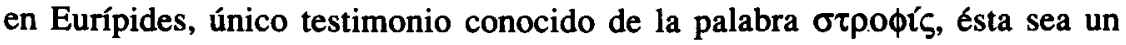
adorno femenino.

Pero el problema principal reside en la interpretación de $\pi \varepsilon ́ p l$, silenciada por Mastrelli. Según comenta Barner es muy improbable, por su posición a principio de verso, que se trate de una preposicion en anástrofe a un término no conservado de la línea anterior ${ }^{9}$. Por ello sugiere de pasada que sería mejor considerarlo un preverbio en tmesis ${ }^{10}$. Realmente el papiro presenta la acentuación $\pi \varepsilon \rho i$, lo cual en mi opinión constituye un apoyo claro a la idea del preverbio en tmesis, ya que la misma acentuación $\pi \grave{\varepsilon} p l$ presenta el papiro en Alc.

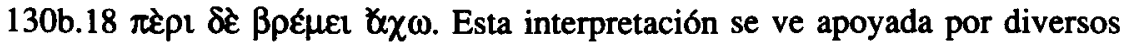
datos léxicos, contextuales y fraseológicos:

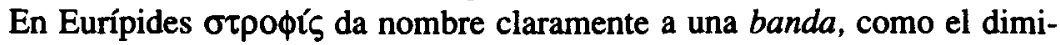

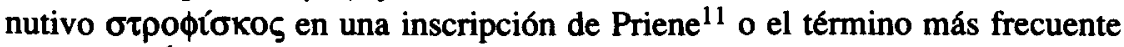
$\sigma \tau \rho \delta \phi o v$. Este denomina la banda que llevan en torno a la cabeza o al pecho

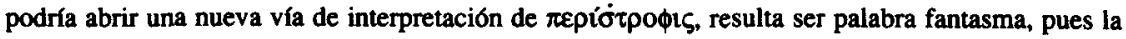
edición de ID 104-26 bis. C .2 lo suprime.

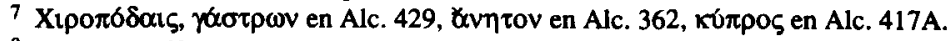

8 Op. cit., p. 68.

9 La anástrofe está documentada en papiros de los lesbios, frente a lo que dice Barner en la

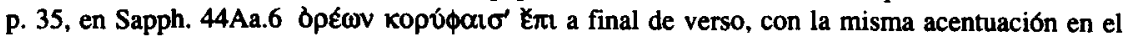
papiro.

10 C. GallavotTI, Saffo e Alceo II, Nápoles $1957^{2}$, p. 85 presenta $\pi \varepsilon \rho i ~ o \tau p o ́ \phi 1 \delta^{\prime}$ [, aunque no comenta el pasaje.

11 IPr. 202.13 (11 a. C.). 
tanto las mujeres como diversos cargos y sacerdotes ${ }^{12}$. Respecto a $\sigma \tau \rho \delta \phi \circ \zeta$, por el contrario, cabe la duda de si en el $h . A p$., Esquilo y Aristófanes significa cinta o cordon, si bien me inclino por lo segundo ${ }^{13}$.

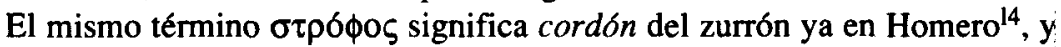
cuerda, soga en general en Hdt. 4.60, IG $2^{2} .1631 .336$ (IV a.C.), Vitr.1.3.6, etc. El mismo sentido tienen $\sigma \tau \rho \circ \phi \varepsilon i o v$ y el compuesto $\pi \varepsilon \rho i \sigma \tau \rho o \phi o \zeta$ en un pasaje de Jenofonte, $X$. Cyn. 2.6, cf. Poll. 5.29.

Como vemos en el primer grupo de palabras, que significan banda o cinta* la noción predominante es la de algo que gira en torno a, es decir que rodea, como $\sigma \tau \rho \varepsilon \pi \tau \sigma \zeta$ collar desde Heródoto ${ }^{15}$. Por el contrario, en los términos que significan cuerda o cordón parece ser la de retorcer ${ }^{16}$. Como sucede con $\sigma \tau \rho \delta \phi o \zeta$ el mismo término puede reflejar, incluso al mismo tiempo, ambas nociones. Si aceptamos, como parece sensato, que el pasaje de Alceo describe una serie de castigos y torturas posibles para el tirano, lo más adecuado es dar a $\sigma \tau \rho \delta \phi ı \varsigma$, , como diminutivo de $\sigma \tau \rho \delta \phi o \zeta$, el significado de cuerda o lazo atado al cual el personaje debería ir andando ( $\phi o i ́ \tau \alpha \nu)$ ante la vista de todos. Lo más probable es que se quisiera poner la cuerda en torno al cuello, lo cual constituye un castigo tradicional ampliamente documentado ${ }^{17}$. Además, disponemos de un paralelo muy cercano, en contexto muy similar, en la misma obra de Alceo; Así, en el comienzo del conocido fragmento 298 , en que antes de narrar el sacrilegio de Ayax el poeta pide castigo para Pítaco, se puede ver que además dẹ

12 Utilizada por mujeres aparece en Pherecr. 106, Ar. Lys. 931, Th. 139, 225, Fr. 647, IQ $2^{2} .1388 .19$, etc.; por sacerdotes en Philoch. 141B, Plu. Arat. 53, Arr. Epict. 3.21. 16, IG 22 1511.3 Ilasos 5.81 (11 a. C.), IG 5(1).1390.179 (Andania I a. C.), $S I G^{3} 869.21$ (Eleusis), etc. y finalmente

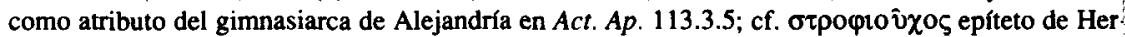
mes en Orph. H. 28.5 .

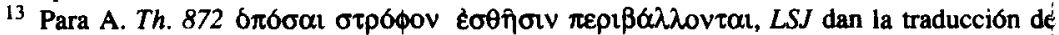
womens' girdle, pero la comparación con $h$. Ap. 122 y 128 hace más bien pensar en un cordóni puesto a la cintura sobre el vestido. En Ar. Fr. 332 Kassel-Austin el sentido es menos claro; En Ai

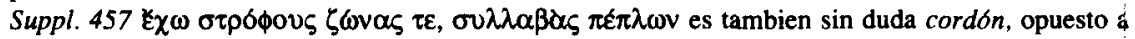
$\zeta \omega v \eta$ cinta, faja, ceñidor, aunque hay quien prefiere la lectura de los manuscritos $\sigma \tau \rho \delta \beta 0 v \zeta$.

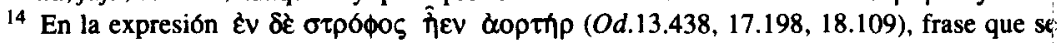
puede entender de dos formas: 1) considerando que otpóфos es substantivo y doptrip tiene fun ción adjetival, significando para colgar (el zurrón); 2) considerando que $\sigma \tau \rho \delta \phi o \zeta$ es adjetivo re torcido y modifica a doptrip correa, cuerda.

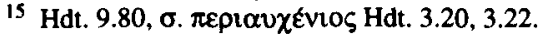

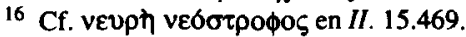

$17 \mathrm{~V}$. últimamente el volumen colectivo Le chátiment dans la cité. Supplices corporels et peine de mort dans le monde antique. Table ronde organisée par l' Ecole française de Rome avec le concours du Centre National de la Recherche Scientifique (Rome 9-11 novembre 1982). Collection de l' Ecole française de Rome 79. Ecole française de Rome, 1984, y en especial la contribución dé N. Loraux, «Le corps étranglé. Quelques faits et beaucoups de représentations», p. 195 ss. 
lapidar al tirano se le quiere ahorcar o echar la soga al cuello ... $\pi \varepsilon \rho \beta \alpha ́ \alpha \lambda o v-$

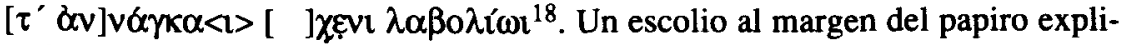
ca $\alpha v \alpha \gamma \gamma \eta$ como $\alpha \gamma \chi \delta ́ v \eta$ soga, pero este sentido se encontraría aislado frente al más abstracto de castigo corporal, violencia, tortura, por lo que, como dice Tarditi, es el nexo con [ $\alpha \zeta] \chi \varepsilon v^{\prime}$ lo que hace que $\alpha v \alpha \gamma \kappa \alpha$ indique la acción de echar la soga al cuello ${ }^{19}$. En el fragmento que discutimos, por el contrario, el

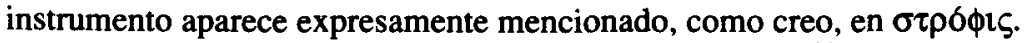

La consideración de $\pi \varepsilon \rho i ́$ como preverbio en tmesis ${ }^{20}$ apoya también esta interpretación, pues para la acción de echar o poner una atadura en torno a una persona o a su cuello, se utilizan normalmente en griego, como vemos también en el fragmento 298 de Alceo, compuestos con preverbio $\pi \varepsilon \rho t-$, siendo los más frecuentes $\pi \varepsilon \rho \imath \beta \alpha \lambda \lambda \omega$ y $\pi \varepsilon \rho \imath \tau i \theta \eta \mu \imath$. Lo más probable es que en algún lugar de la parte perdida tras $\sigma \tau \rho \sigma \phi 1 \delta^{\prime}$ apareciera una forma simple de $\beta \alpha \lambda \lambda \omega$ o quizá de $\tau \dot{\theta} \theta \eta \mu \mathrm{t}$. Se pueden aducir diversos paralelos: A. Pr. $52 \tau \hat{\omega} \delta \varepsilon \delta \varepsilon \sigma \mu \alpha$ $\pi \varepsilon \rho \imath \beta \alpha \lambda \varepsilon \hat{\imath} v$, E. $B a .1021 \hat{\omega}$ B $\alpha \kappa \chi \varepsilon, \theta \eta \rho \alpha \gamma \rho \varepsilon v \tau \hat{\alpha}$ B $\alpha \kappa \chi \alpha \hat{\alpha}$... $\pi \varepsilon \rho \hat{\imath} \beta \alpha \lambda \varepsilon$

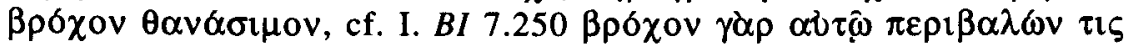
$\pi \delta \rho \rho \omega \theta \varepsilon v \mathcal{\varepsilon} \mu \varepsilon \lambda \lambda \varepsilon v \varepsilon \dot{\varepsilon} \tau \sigma \pi \alpha \sigma \varepsilon \iota v$. En estos tres ejemplos el instrumento utilizado va en acusativo, constituyendo el complemento directo del verbo. En otra construcción frecuente el instrumento aparece en dativo instrumental: Hdt. 4.60 , al describir los sacrificios entre los escitas, utiliza la expresión $\beta \rho \delta \chi \omega$

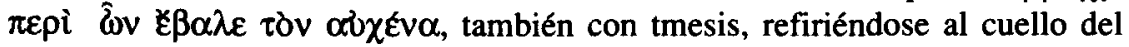

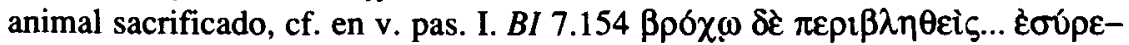

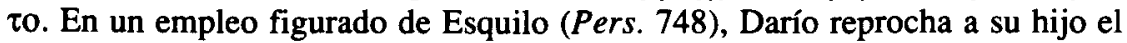
haber intentado someter al Helesponto como a un esclavo, $\pi \varepsilon \delta \alpha \iota \varsigma \sigma \phi v \rho \eta \lambda \alpha-$

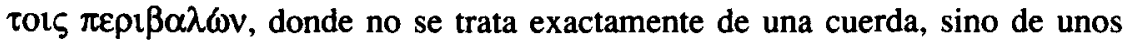
grilletes. En realidad el verbo $\pi \varepsilon \rho \iota \beta \alpha \lambda \lambda \omega \omega$ se utiliza por extensión con otro tipo de instrumentos de castigo o con abstractos designando el castigo, como sucede en Alc. 298.2. Por su parte el verbo $\pi \varepsilon \rho \imath \tau i \theta \eta \mu$ se documenta escasamente con complemento directo de instrumentos de castigo ${ }^{21}$, pero es muy frecuente-

18 Así en la edición de Voigt. En el v. $3[\alpha \zeta] \chi £ v ı$ parece el suplemento más probable, si bien hay quien ve problemas por el hiato con el final del verso anterior. Cf. A.M. van ERP TAALMAN KIP, "Aiax and Kassandra», en A.M. van ERP TAalman KIP, J.M. BREMER, S.R. SLINGS (edd.), Some recently found Greek poems. Text and Commentary. Mnemosyne Suppl. 99, Leiden 1987, p. 106.

19 G. TARDITI, «L' $\alpha \sigma e \hat{\beta \varepsilon 1} \alpha$ di Aiace e quella di Pittaco». $Q U C C$ 8, 1969, p. 90. Cf. tambien la editio princeps de R. MERKELBACH, «Ein Alkaios-Papyrus». ZPE 1, 1967, p. 81 ss., y vaN ERP TAALMAN KIP, en op. cit, p. 105 ss.

20 La tmesis es frecuente en Alceo: 10.4, 70.9, 129.3, 140.11, 298.26, 338.5, 338.6, 346.2, 359.4, 362.3, 367.2, 376, 377.

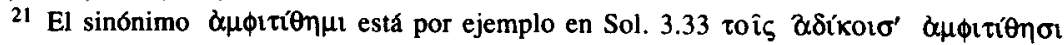
$\pi \varepsilon \delta \alpha \varsigma$. 
mente utilizado, como en los mismos poetas lesbios, para la acción de poner o ponerse coronas o collares en torno al cuello: Alc. $362.2 \pi \varepsilon \rho i$ $\tau \alpha i \zeta ~ \delta \varepsilon \rho \alpha \imath \sigma<\uparrow>$

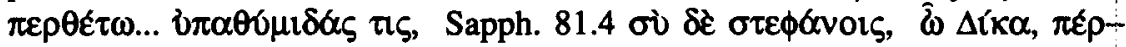

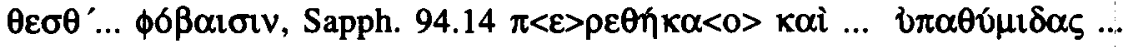
$\alpha \mu \phi^{\prime} . . . \delta \varepsilon \rho \alpha$.

En nuestro fragmento $\sigma \tau \rho \delta \phi_{1} \delta^{\prime}$ esconde seguramente un acusativo, aunque no se puede desechar la posibilidad del dativo. El paralelo de Alc. 298 po: co puede ayudar, pues el estado fragmentario del papiro impide ver con claridad la construcción sintáctica, y se duda entre recorstruir un dativo

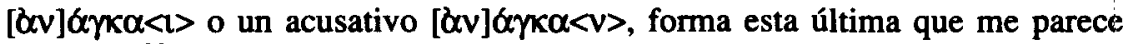
preferible $^{22}$.

22 Desde la edición de Merkelbach se suele preferir el dativo. El acusativo, propuesto por W. LUPPE ( $\alpha^{\prime} A v a ́ x \kappa \alpha$ im Kölner Alkaios», ZPE 33, 1979, p. 29 s.), por un lado evitaŕa el hiato en-

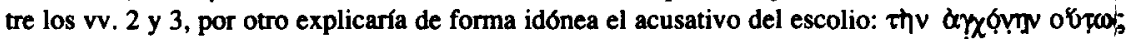

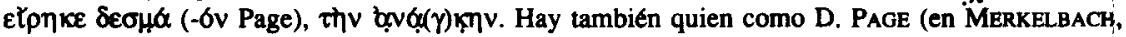

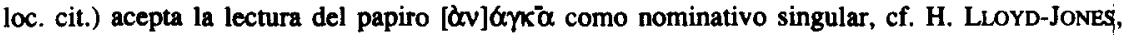
"The Cologne Fragment of Alcaeus», GRBS 9, 1968, p. 125 ss., y C. GALl.AvOTT, «Riconstruzione del nuovo carme di Alceon, $Q U C C$ 8, 1969, p. 83 ss. Sobre todo ello v. van ERP TAALMAN KIP, ari. cit., p. $106 \mathrm{~s}$. 